\title{
Effects of condensed tannins on established populations and on incoming larvae of Trichostrongylus colubriformis and Teladorsagia circumcincta in goats
}

\author{
Virginie PAOLINI ${ }^{\mathrm{a} *}$, Audrey FRAYSSINES $^{\mathrm{a}}$, France DE LA FARGE $^{\mathrm{b}}$, \\ Philippe DORCHIES ${ }^{\mathrm{a}}$, Hervé HOSTE ${ }^{\mathrm{a}}$

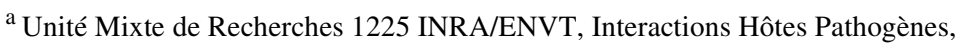 \\ École Nationale Vétérinaire de Toulouse, 23, chemin des Capelles, 31076 Toulouse, France \\ ${ }^{\mathrm{b}}$ Laboratoire de Biochimie, Faculté de Médecine de Rangueil, 31403 Toulouse, France
}

(Received 16 September 2002; accepted 20 December 2002)

\begin{abstract}
The use of tanniferous plants or tannins represents one alternative approach to the control of gastrointestinal parasites in ruminants but most data have been obtained in sheep. The current study was therefore performed in goats with two objectives: firstly, to investigate the effects of condensed tannins (CT) on adult populations of Trichostrongylus colubriformis and Teladorsagia circumcincta; secondly, to examine their effects on the establishment of infective larvae of these two species. In experiment 1 , two groups of kids were infected with $6000 \mathrm{~L} 3$ of $T$. colubriformis and $6000 \mathrm{~L} 3$ of $T$. circumcincta. After 7 weeks, quebracho extracts were administered per os for 8 days to one group. A comparable group which did not receive tannins was included as the control. The kids were slaughtered on week 11. Parasitological and pathophysiological parameters were measured weekly. Worm counts were assessed and mast cells, globule leukocytes and eosinophils were counted in the abomasal and intestinal mucosae. Tannin administration was associated with a decrease in egg excretion, and a decrease in female fecundity, but with no changes in worm numbers. These changes were associated with an increased number of intestinal mast cells. In experiment 2, 24 goats were used according to a $2 \times 2$ factorial design, depending on infection and tannin administration. Two groups were either infected with $6000 \mathrm{~L} 3$ of T. colubriformis or T. circumcincta. Within each group, the goats were either drenched or undrenched with tannin extracts. Pathophysiological parameters were measured weekly. Twelve days after the cessation of tannin administration, the goats were slaughtered. Worm counts and female worm fecundity were determined. Tannin consumption was associated with a significant reduction $(P<0.01)$ of Trichostrongylus populations and a close to significant reduction for Teladorsagia. No effect on fecundity was observed. Our results (1) confirm the consequences of condensed tannins on nematodes in goats as in sheep and (2) indicate divergent effects depending on the parasitic stage exposed to the condensed tannins.
\end{abstract}

Trichostrongylus colubriformis / Teladorsagia circumcincta / goat / tannin / alternative methods of control

* Correspondence and reprints

Tel.: (33) 5611938 75; fax: (33) 5611939 44; e-mail: v.paolini@envt.fr 


\section{INTRODUCTION}

Gastrointestinal parasitism with nematodes remains a major threat to an efficient production in small ruminants. Up to now, the control of these parasitic infections has been largely based on the repeated use of anthelmintics. Due to the increasing demand of the consumers to limit the use of chemical substances in farming and because of the development of anthelmintic resistance in different worm species [10], there is currently a need to seek alternative or complementary solutions to the control of gastrointestinal parasitism [8, 29]. Amongst these alternative methods, the use of tanniferous plants has been explored in several studies. The initial results were obtained in New-Zealand. Niezen et al. [18-21] from field studies suggested that the consumption of tanniferous forages could affect the biology of the gastrointestinal worms, mainly by decreasing egg excretion, and therefore could contribute to modulate the epidemiology of these parasitic diseases. Further experimental in vivo studies, using quebracho extracts (a rich source of condensed tannins), tended to confirm the initial results obtained with the tanniferous plants [1-3]. In addition, some recent in vitro experiments have also ascertained an effect of condensed tannins on gastrointestinal nematodes [3, 17].

The vast majority of the in vivo experimental studies on the effects of tannins on worm populations have been performed in sheep. In contrast, few data are available on goats [12], despite their differences in feeding behaviour, in metabolism and in digestive physiology [7] and their better adaptation to exploit tanniferous plants $[27,28]$. It can be postulated that such physiological, metabolic and immunological peculiarities could strongly modulate the interactions between tannins and parasites compared to sheep. Therefore direct studies on goats are needed.

The objectives of the current study were to assess in controlled conditions the effects of condensed tannins on nematode populations in goats, in order to compare the results with previous data obtained in sheep. Because of their high prevalence and their pathological consequences in goats, Trichostrongylus colubriformis and Teladorsagia circumcincta were used as parasitic models of infection. We compared the effects of condensed tannins either on an established population of adult worms or on incoming third-stage larvae. Therefore, two different experiments were performed: one using tannins as a curative treatment, and one using tannins given in a preventive mode, concomitantly with the larval dose. The source and concentration of tannins were chosen in order to facilitate comparisons with studies previously performed in sheep [1-3].

\section{MATERIALS AND METHODS}

\subsection{Experimental design}

\subsubsection{Experiment 1: \\ Curative administration of condensed tannins}

Thirteen naïve, four-month-old kids were experimentally infected (D0) with 6000 infective larvae (L3) of Trichostrongylus colubriformis and 6000 infective larvae of Teladorsagia circumcincta (D0). The third-stage larvae were obtained from donor sheep infected with $T$. colubriformis or $T$. circumcincta. The kids were reared indoors to exclude natural nematode infection. They were offered a high quality pelleted food, and gramineous hay.

Seven weeks after infection, at D51, the animals were divided into two groups, balanced according to sex, bodyweight and the level of egg excretion. Seven kids composed the tannin group. They received daily, per os, for eight days, from D51 to D59 condensed tannins from quebracho extracts (from the bark of the tropical tree Schinopsis spp.). These extracts were diluted in $150 \mathrm{~mL}$ of water at $25{ }^{\circ} \mathrm{C}$ and 
were given orally five times per day. They represented $5 \%$ of the DM diet of the kids. The 6 remaining animals composed an infected control group, which received daily, for eight days, at the same period, an equal volume of water. All the kids were slaughtered 18 days after the end of the tannin administration, on D77.

\subsubsection{Experiment 2: Preventive administration of condensed tannins}

Twenty-four culled, naïve, Saanen goats, were used. The goats were reared indoors to exclude natural nematode infection. They were offered a high quality pelleted food, and gramineous hay. They were divided into 4 groups of 6 goats according to their bodyweight. Groups 1 and 2 were experimentally infected for three consecutive days (D0, D1, D2) with 2000 thirdstage larvae of $T$. colubriformis per day. Groups 3 and 4 were experimentally infected with $T$. circumcincta according to the same experimental design. Groups 1 and 3 received daily, for 8 days (D-3 to D4), condensed tannins (three times per day).The quebracho extracts were similar to those used in the first experiment. They represented $5 \%$ of the DM diet of the animals. All the goats were slaughtered 12 days after the end of the tannin administration, on D16.

\subsection{Parasitological techniques}

\subsubsection{Experiment 1: Curative administration of condensed tannins}

Faecal samples were collected from the rectum twice per week to measure nematode egg excretion. The egg counts were performed according to a modified $\mathrm{Mc}$ Master technique [24]. At necropsy, the abomasa and the small intestine were removed and the worms were recovered from both luminal contents and digesta of either the abomasal or intestinal mucosa after $5 \mathrm{~h}$ incubation in saline at $37^{\circ} \mathrm{C}$. Total worm burdens were estimated from $10 \%$ aliquots of lumen and mucosal digest for each goat. The stage and sex of $T$. colubriformis and $T$. circumcincta were identified according to Douvres [6] and Rose [26]. For the determination of the number of eggs in the uterus of female worms (fecundity in utero), 20 females per kid were separated at random from each aliquot and cleared in lactophenol.

\subsubsection{Experiment 2: Preventive administration of condensed tannins}

At necropsy, the small intestine (in groups 1 and 2) and the abomasa (in groups 3 and 4) were collected to determine the number of parasites from both the lumen and the mucosa based on a $10 \%$ aliquot method as previously described. The stage and sex of T. colubriformis and T. circumcincta were identified as previously described. For determination of the number of eggs in the uterus of the female worms (fecundity in utero), 10 females per kid were separated at random from each aliquot and cleared in lactophenol.

\subsection{Blood analyses}

\subsubsection{Experiment 1: Curative administration of condensed tannins}

Blood samples were collected weekly by venipuncture into vacuum tubes and heparinised tubes. They were used to measure the number of blood eosinophils, the serum concentrations of pepsinogen and inorganic phosphate. Eosinophil counts were performed using Fast-Read slides ${ }^{\circledR}$ and Carpentier solution according to Dawkins et al. [5]. Pepsinogen concentrations were measured according to the method described by Kerboeuf [14]. Inorganic phosphate concentrations were 
determined according to the method described by Robinson et al. [25].

\subsubsection{Experiment 2: Preventive administration of condensed tannins}

Blood samples were collected weekly by venipuncture into vacuum tubes. They were used to dose the serum concentrations of inorganic phosphate (in groups 1 and 2) and serum concentrations of pepsinogen (in groups 3 and 4) according to the same techniques as described in Experiment 1.

\subsection{Histological analyses}

\subsubsection{Experiment 1: Curative administration of condensed tannins}

At necropsy, histological samples were collected from both fundus and pylorus and from the small intestine for determination of the number of mast cells, globule leukocytes and eosinophils in the mucosae according to the technique described by Larsen et al. [15] and Huntley et al. [9]. The stained cells were enumerated at a 400 magnification using a calibrated graticule encompassing an area of $0.25 \mathrm{~mm}^{2}$. Mean cell densities for each tissue and each cell type were assessed from counts on 10 fields, which were randomly selected. The results are expressed as the mean number of cells per $\mathrm{mm}^{2}$ of mucosa.

\subsection{Statistical analyses}

In both experiments, before analysis, data for egg counts, worm numbers and cell counts were $(\log (x+1))$ transformed to normalise their distribution.

Data on worm burden, fecundity in utero and histological counts (only in Experiment 1) were compared between the different groups using the non parametric Mann and Whitney test. For the other measurements (faecal egg counts and pathophysiological parameters), the comparisons were performed using a repeated measure analysis of variance (SYSTAT 9.0 software for Windows 1998, SPSS Inc. Chicago, USA). For the first experiment, two periods were considered. The first period corresponded to the dates before the administration of quebracho extracts (D0 to D51). The second period included the period of tannin administration (D51 to D59) plus the 18 days between the cessation of tannins and the end of the experiment (D60 to D77). For the second experiment, the comparisons were performed on data collected on each sampling date.

\section{RESULTS}

\subsection{Curative administration of condensed tannins (Experiment 1)}

\subsubsection{Faecal egg counts (FECs) (Fig. 1)}

Before quebracho distribution (D0 to D51), no significant difference in egg excretion (Fig. 1) was observed between the two groups. In contrast, during the second period, starting with the tannin administration, FECs were lower in kids consuming the quebracho extracts and the difference between both groups was close to significance $(P<0.06)$. This reduction persisted after the quebracho drench was stopped. Overall, the egg excretion was $50 \%$ lower in the tannin group compared to the control group.

\subsubsection{Worm burden and fecundity (Tab. I)}

Concerning the worm burdens, no significant differences were observed for the two species of nematodes between the treated and the control groups (Tab. I). However, the fecundity in utero of the female worms in the tannin group was significantly reduced $(P<0.05)$ for the intestinal species $T$. colubriformis (Tab. I). 


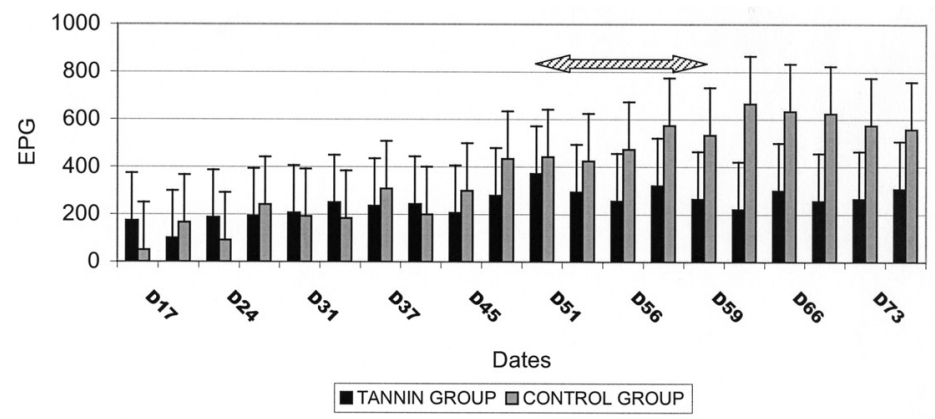

Figure 1. Arithmetic mean faecal egg counts (eggs per gram \pm S.D.) in the tannin and the control groups before and after administration of tannins. A difference $(P<0.06)$ was observed between both groups from D51 to D77. The arrow represents the 8-day period of administration of quebracho extracts (D51 to D59).

Table I. Mean worm burden $( \pm$ S.D. $)$, sex ratio and fecundity in utero from each species of nematodes for the tannin and the control groups. * indicates a significant difference $P<0.05$ and ** $P<0.01$.

\begin{tabular}{lcccc}
\hline Experiment 1 & T. colubriformis & T. colubriformis & T. circumcincta & T. circumcincta \\
\hline & Tannin group & Control group & Tannin group & Control group \\
Worm burden & $358( \pm 237)$ & $133( \pm 66)$ & $441( \pm 390)$ & $515( \pm 463)$ \\
Sex ratio & 0.91 & 1.21 & 0.9 & 1.28 \\
Fecundity in utero & $21.2^{*}$ & 23.9 & 53.1 & 56.6 \\
\hline Experiment 2 & \multicolumn{5}{|c}{} \\
\hline Worm burden & $981.7( \pm 627)^{* *}$ & $2756( \pm 908)$ & $114( \pm 137)$ & $385( \pm 224)$ \\
Sex ratio & 1.09 & 0.98 & 1.08 & 1.26 \\
Fecundity in utero & 21.9 & 19.9 & 34.3 & 35.4 \\
\hline
\end{tabular}

Similarly for the abomasal species, the females in the treated group were less fecund compared to those from the controls but the difference was not significant.

\subsubsection{Pathophysiological measurements}

Irrespective of the experimental period (before or after the administration of quebracho extracts), there was no significant difference between the two groups for the concentrations of pepsinogen and inorganic phosphate (data not shown).

In addition, no significant difference was observed for the blood eosinophils between the treated and the control groups, during the whole study (data not shown).

\subsubsection{Mucosal cellular response (Fig. 2)}

In the abomasal sites (fundus and pylorus) (Fig. 2), there were no significant differences between the tannin and the control group for any of the three cell types. In the small intestine (Fig. 2), higher numbers of mast cells and eosinophils were found in the tannin group compared to the untreated group, but the difference was only significant for mast cells $(P<$ 0.02). No significant difference was observed for globule leukocytes between the two groups of animals. 


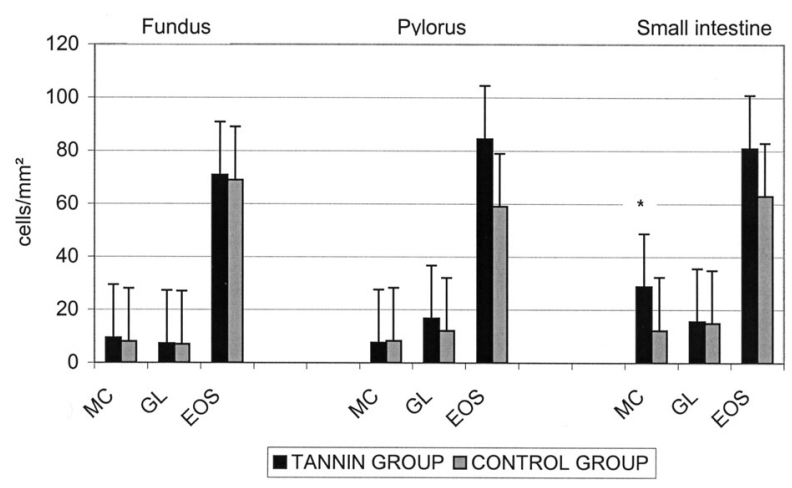

Figure 2. Arithmetic mean $( \pm$ S.D. $)$ numbers of inflammatory cells [mast cells $(\mathrm{MC})$, globule leukocytes (GL), eosinophils (EOS)] in the mucosae of fundus, pylorus and small intestine in the two experimental groups. * indicates a significant difference $P<0.02$.

\subsection{Preventive administration of condensed tannins (Experiment 2)}

\subsubsection{Worm burden and fecundity (Tab. I)}

\section{T. colubriformis:}

The total worm number recovered in the tannin group was significantly reduced $(P<0.01)$ compared to the controls (Tab. I). However, the sex ratios were similar in both groups. Concerning the fecundity in utero of the female worms, no significant difference was observed between the two groups (Tab. I).

\section{T. circumcincta:}

The worm burden was lower in the tannin group than in the control group (Tab. I) and the difference was close to significance $(P<0.08)$. Both groups had similar sex ratios and fecundity in utero (Tab. I).

\subsubsection{Pathophysiological measurements}

For both concentrations of inorganic phosphate and pepsinogen (data not shown), no significant difference was observed between the groups receiving tannins and those corresponding to infected controls.

\section{DISCUSSION}

Three main results were acquired in the current study. Firstly, the data confirmed that condensed tannins do affect adult worm populations in goats as previously demonstrated in sheep; secondly, quebracho extracts also have an impact on the early establishment of third-stage larvae and lastly, the effects of condensed tannins on worm biology depend on the timing of administration in relation to the parasitic cycle.

The main result of the curative experiment was the decrease in egg excretion observed in the tannin group. This reduction started immediately after the distribution of quebracho and represented an overall $50 \%$ decrease compared to the controls. Under similar conditions of infection, a decrease in faecal egg counts has also been observed in goats infected with Haemonchus contortus [22]. In addition, similar 
results have also been observed in naturally infected goats that received sainfoin hay, rich in tannins [23]. These results are also supported by data from sheep experimentally infected with $T$. colubriformis and treated with quebracho extracts $[1,2]$. In these studies, the level of reduction in egg output after tannin administration was comparable to that observed in the current study in goats. In addition, our results also indicate that the effect of condensed tannins on worm populations can be persistent since the reduction in egg output was still present even 18 days after the cessation of quebracho administration. This is the first time this effect has been reported in goats. Niezen et al. [20] observed a similar persistent effect in lambs fed Sulla (Hedysarum coronarium), which is a tanniferous plant. The persistent impact of the condensed tannins appear to be present both in goats and sheep. From the current results, it is difficult to assess whether this persistency is related to irreversible lesions induced to female worms or whether this is due to a lasting stimulation of the local immune response. However, whatever the underlying mechanism, this delayed effect needs to be confirmed in further experiments since it could have major implications in modulating the epidemiology of nematode infections.

In sheep, the effects of different concentrations of tannins have been measured, showing that at concentrations exceeding $16 \%$, clinical signs appear [3]. Similar data are still lacking in goats but it would be worth investigation particularly to compare the physiological response in sheep and goats considering the better adaptation of this latter host species to the use of tanniferous plants.

The decrease in faecal egg counts could have a double origin. This could either be a consequence of a diminution in the worm burden, or a reduction in the fecundity of female worms.

No difference in worm counts was observed between the two experimental groups for both species. This result was also confirmed by the lack of major differences in the pathophysiological parameters, which are related to the two nematode populations. These observations suggested, in goats, an absence of an effect of tannins, at the applied concentration, on worm numbers after they have been established. Similarly, a lack of an effect of tannins on adult worms was also observed in goats infected with $H$. contortus [22]. In contrast, in sheep, the effect of tannins on worm populations varied according to the nematode species. A reduction was observed in sheep infected with T. colubriformis receiving a $8 \%$ quebracho concentration [3] but, at the same concentration, there was no effect on $H$. contortus or T. circumcincta [3].

Concerning the fecundity in utero of female worms, a decrease was recorded for both species, although the difference was significant only for T. colubriformis. Similarly, a reduced fecundity per capita was also observed for female $H$. contortus, in goats treated with quebracho extracts [22]. In sheep, the fecundity per capita of female T. colubriformis was also modified after tannin consumption [2, 3]. From the current results, it appears that the decrease in egg output observed after the administration of quebracho tannins to goats infected with an established population did not relate to a reduction in worm counts but was more associated with a reduced fecundity of female worms. The mechanism remains unclear but a possible effect of tannins on the female genital tract will be explored.

A curative effect of condensed tannins on gastrointestinal nematodes, similar to those observed in sheep, was confirmed in goats. In contrast, very few data are available on the possible influence of condensed tannins on incoming larvae. The main result from the preventive study was a reduction by two thirds of the worm populations recovered at necropsy, this effect being significant for the intestinal 
species and close to significance for the abomasal one. This result was not associated with any change in female worm fecundity. In sheep, a decrease in $T$. circumcincta populations was associated with the consumption of sulla, but not for T. colubriformis [20]. However, the worm fecundity was not measured in this study.

The possible mechanisms responsible for the effects of tannins on nematode parasites of ruminants, either the adult or infective larval stage, remain hypothetical. A direct effect has been suggested, which is supported by the results of in vitro tests $[3,13,17]$. The results from the preventive study also supported this hypothesis because of the experimental design, allowing a short delay between the time of infection and the death of the goats.

However, an indirect mechanism has also been evoked. Because tannins protect proteins against ruminal degradation [11, 16], their consumption could stimulate the host immune response [4]. Nevertheless, evidence to support this hypothesis remains scarce. To our knowledge, the current experiment is the first one where a significant increase in mucosal mast cells was observed in relation to tannin administration. During the previous study on $H$. contortus, infection in goats drenched with quebracho extracts [22], an increased density of the three main inflammatory cell types, i.e. mast cells, globule leukocytes and eosinophils, was also observed in the fundus but the difference was not significant.

In conclusion, the comparison of the results from the two experiments comprising the current study indicate that, in goats, the effects of condensed tannins on nematodes strongly depend on the parasitic stage present at the time of administration. It appears that tannins can affect the establishment, and consequently reduce the worm burden when acting on third stage larvae. In contrast, they seem to mainly affect the worm reproductive function and the consequent contamination of pasture in animals harbouring adult worms. In comparison, in sheep, a reduction of abomasal worm burdens has been observed irrespective of the timing of tannin administration.

These effects could have major consequences on the contamination of pastures and offer an alternative approach to controlling gastrointestinal parasitism in small ruminant farming.

\section{ACKNOWLEDGMENTS}

The authors would like to thank the financial support from the European Union through the Contract WORMCOPS (number QLK5-CT 2001-01843) which is part of a collaboration between Denmark, UK, the Netherlands, Sweden, Spain and France. Mrs Grisez, Mrs Prevot, Mr Bergeaud and Mr Terrancle are also thanked for their technical assistance.

\section{REFERENCES}

[1] Athanasiadou S., Kyriazakis I., Jackson F., Coop R.L., Effects of short term exposure to condensed tannins on adult $T$. colubriformis, Vet. Rec. 146 (2000) 728-732.

[2] Athanasiadou S., Kyriazakis I., Jackson F., Coop R.L., Consequences of long-term feeding with condensed tannins on sheep parasited with $T$. colubriformis, Int. J. Parasitol. 30 (2000)1025-1033.

[3] Athanasiadou S., Kyriazakis I., Jackson F., Coop R.L., Direct anthelmintic effects of condensed tannins towards different gastrointestinal nematodes of sheep in vitro and in vivo studies, Vet. Parasitol. 99 (2001) 205-219.

[4] Coop R.L., Kyriazakis I., Influence of host nutrition on the development and consequences of nematode parasitism in ruminants, Trends Parasitol. 17 (2001) 325-330.

[5] Dawkins H.J.S., Windon R.G., Eagleson G.K., Eosinophil responses in sheep selected for high and low responsiveness to T. colubriformis, Int. J. Parasitol. 19 (1989) 199-205.

[6] Douvres F.W., The morphogenesis of the parasitic stages of $T$. axe $i$ and T. colubriformis, nematode parasites of cattle, Proc. Helm. Soc. Washington 24 (1957) 4-14. 
[7] Hoste H., Chartier C., Résistance des chèvres aux strongyloses gastrointestinales : différences avec les moutons, Le Point Vétérinaire 29 (1998) 69-74.

[8] Hoste H., Chartier C., Le Frileux Y., Control of gastrointestinal parasitism with nematodes in dairy goats by treating the host category at risk, Vet. Res. 33 (2002) 531-546.

[9] Huntley J.F., Patterson M., McKellar A., Jackson F., Stevenson L.M., Coop R.L., A comparison of the mast cell and eosinophil responses of sheep and goats to gastrointestinal nematode infections, Res. Vet. Sci. 58 (1995) 5-10.

[10] Jackson F., Coop R.L., The development of anthelmintic resistance in sheep nematodes, Parasitology 120 (2000) 95-107.

[11] Jean-Blain C., Aspects nutritionnels et toxicologiques des tanins, Rev. Méd. Vét. 149 (1998) 911-920.

[12] Kabasa J.D., Opuda-Asibo J., Ter Meulen U., The effect of oral administration of polyethylene glycol on faecal helminth egg counts in pregnant goats grazed on browse containing condensed tannins, Trop. Anim. Health Prod. 32 (2000) 73-86.

[13] Kahn L.P., Diaz-Hernandez A., Tannins with anthelmintic properties, Proc. Int. Workshop, Adelaide, Australia, ACIAR Proceedings 92 (2000) 130-138.

[14] Kerboeuf D., Changes in pepsinogen, proteins and lipids in the serum during experimental haemonchosis in sheep, Ann. Rech. Vet. 8 (1977) 257-266.

[15] Larsen J.W.A., Anderson N., Vizard A., Anderson G.A., Hoste H., Diarrhoea in Merino ewes during winter: association with trichostrongylid larvae, Aust. Vet. J. 71 (1994) 365-372.

[16] Mangan J.L., Nutritional effects of tannins in animal feeds, Nutr. Res. Rev. 1 (1988) 209231.

[17] Molan A.L., Alexander R.A., Brookes I.M., Mac Nabb W.C., Effect of an extract from Sulla (Hedysarum coronarium) containing condensed tannins on the migration of three sheep gastrointestinal nematodes in vitro, Proc. N. Z. Soc. Anim. Prod. 60 (2000) 21-25.

[18] Niezen J.H., Robertson H.A., Waghorn G.C., Charleston W.A.G., Production, faecal egg counts and worm burdens of ewe lambs which grazed six contrasting forages, Vet. Parasitol. 80 (1998) 15-27.
[19] Niezen J.H., Waghorn G.C., Charleston W.A.G., Establishment and fecundity of Ostertagia circumcincta and Trichostrongylus colubriformis in lambs fed Lotus $(L$. pedunculatus) or perennial ryegrass (Lolium perenne), Vet. Parasitol. 78 (1998) 13-21.

[20] Niezen J.H., Charleston W.A.G., Robertson H.A., Shelton D., Waghorn G.C., Green R., The effect of feeding sulla (Hedysarum coronarium) or lucerne (Medicago sativa) on lamb parasite burdens and development of immunity to gastrointestinal nematodes, Vet. Parasitol. 105 (2002) 229-245.

[21] Niezen J.H., Waghorn G.C., Graham T. Carter J.L., Leathwick D.M., The effect of diet fed to lambs on subsequent development of Trichostrongylus colubriformis larvae in vitro and on pasture, Vet. Parasitol. 105 (2002) 269-283.

[22] Paolini V., Athanasiadou S., Dorchies Ph., Hoste H., Effets des tannins condensés et des plantes à tanins sur le parasitisme gastrointestinal par les nématodes chez la chèvre, $9^{\mathrm{e}}$ Rencontre Recherche Ruminants, Paris, 4-5 décembre 2002.

[23] Paolini V., Dorchies Ph., Hoste H., Effects of sainfoin hay on gastrointestinal infection with nematodes in goats, Vet. Rec. (to be published).

[24] Raynaud J.P., Étude de l'efficacité d'une technique de coproscopie quantitative pour le diagnostic de routine et le contrôle des infestations parasitaires des bovins, ovins, équins et porcins, Ann. Parasitol. Hum. Comp. (Paris) 45 (1970) 321-342.

[25] Robinson R., Roughan M.E., Wagstaff D.F., Measuring inorganic phosphate without using a reducing agent, Ann. Clin. Biochem. 8 (1971) 168-170.

[26] Rose J.H., The development of the parasitic stages of Ostertagia ostertagi, J. Helminthol. 43 (1969) 173-184.

[27] Silanikove N., The physiological basis of adaptation in goats to harsh environments, Small Rum. Res. 35 (2000) 181-193.

[28] Silanikove N., Gilboa N., Perevolotsky A., Nitsan Z., Goats fed tannin-containing leaves do not exhibit toxic syndromes, Small Rum. Res. 21 (1996) 195-201.

[29] Waller P.J., International approaches to the concept of integrated control of nematode parasites of livestock, Int. J. Parasitol. 29 (1999) 155-164. 